\title{
Organotopic organization of the cervical vagus nerve
}

Nicole Thompson ${ }^{1 * \dagger} \uparrow$, Enrico Ravagli ${ }^{1} \uparrow$, Svetlana Mastitskaya ${ }^{1} \uparrow$, Francesco Iacoviello $^{2}$, ThaleiaRengina Stathopoulou ${ }^{3}$, Justin Perkins ${ }^{3}$, Paul R. Shearing ${ }^{2}$, Kirill Aristovich ${ }^{1}$, David Holder ${ }^{1}$

${ }^{1}$ Department of Medical Physics and Biomedical Engineering, University College London; Gower Street, London WC1E 6BT, United Kingdom

${ }^{2}$ Department of Chemical Engineering, University College London; Gower Street, London WC1E 6BT, United Kingdom

${ }^{3}$ Department of Clinical Science and Services, The Royal Veterinary College; Hawkshead Lane, Hatfield AL9 7TA, United Kingdom

*Corresponding author. Email: nicole.thompson@ucl.ac.uk

$\dagger$ These authors contributed equally to this work

\section{Abstract:}

The functional anatomy of fascicles evident in human and large mammal cervical vagus nerve is unknown. Organ-specific organization in the pig cervical nerve has been observed for cardiac, recurrent laryngeal and pulmonary function using anatomical tracing with X-ray microCT, and functional connectivity with selective stimulation or fast neural Electrical Impedance Tomography (EIT) with a custom nerve cuff. Electrical stimulation of the vagus nerve (VNS) is currently used to treat drug-resistant epilepsy and depression but current practice of stimulation of the entire nerve causes undesired side effects. These findings pave the way for improved outcomes in VNS as unwanted side effects could be reduced by targeted selective stimulation of identified organspecific fascicles.

\section{One-Sentence Summary:}

Parasympathetic innervation to the heart, lungs and larynx is organized in an organ-specific way in the cervical vagus nerve. 


\section{Main Text:}

The functional anatomy of somatic peripheral nerves has been well-studied with serial histological tracing. It has been shown that fascicles observed on a nerve cross-section map reasonably logically to supplied dermatomes and muscle groups $(1-3)$. The human vagus nerve is the main peripheral nerve of the autonomic nervous system (ANS) and provides innervation to about eight visceral organs in the thorax and abdomen as well as the larynx. It may contain up to 21 fascicles at the cervical level $(4,5)$, but, in contrast to the somatic case, their anatomical relation to supplied organs and function is almost entirely unknown (6-8). By homology to the somatic nervous system, it seems reasonable to postulate that fascicles are arranged according to their supply to individual organs and possibly specific functions.

Elucidation of this could be of value in treatment of medical conditions in the field of "Electroceuticals" - electrical modulation of the ANS (9), such as vagus nerve stimulation (VNS). This is currently accomplished with stimulation of the entire cervical vagus nerve; this indiscriminately modulates all organs supplied and consequent unwanted side-effects, such as hoarseness during VNS for epilepsy, limit therapeutic efficacy (10-12). In principle, this could be avoided by spatially-selective stimulation of individual fascicles. Determination of their functional anatomy might provide the key to improvement of VNS with selective stimulation and so avoidance of unwanted side-effects.

The purpose of this study was to determine the functional anatomy of fascicles in the left cervical vagus of the pig in relation to cardiac, pulmonary, and laryngeal function. Fast neural Electrical Impedance Tomography (EIT) is a novel imaging method which enables tomographic imaging of localized compound action potential activity in fascicles within peripheral nerve. It employs a flexible silicone rubber cuff with 14 electrodes and averaging to a repeated trigger, such as the ECG, respiration, or electrically stimulated compound action potentials (8). The functional anatomy was determined with fast neural EIT as well as trial-and-error selective electrical stimulation using flexible nerve cuffs in vivo. These functional measures were validated by ex vivo anatomical tracing of fascicles identified in the cervical vagus nerve from their peripheral organ branches, using microCT with iodine staining. 
bioRxiv preprint doi: https://doi.org/10.1101/2022.02.24.481810; this version posted February 25, 2022. The copyright holder for this preprint

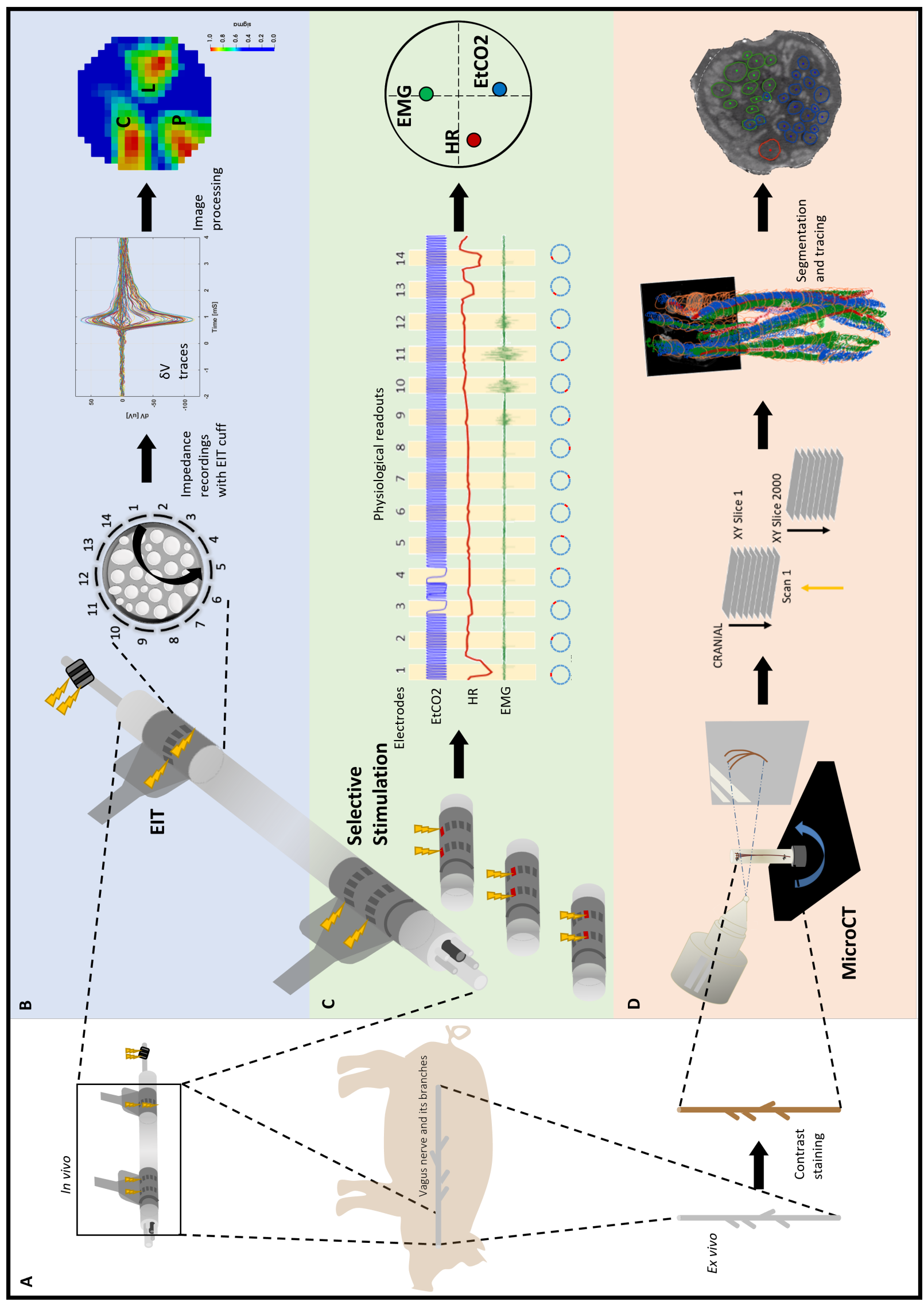


Fig. 1: Experimental design for pig cervical vagus nerve imaging with Electrical Impedance Tomography (EIT), selective stimulation (SS), and micro-computed tomography (microCT). (A) in vivo experiment in pigs with EIT and SS cuffs placed around the left vagus nerve $(\mathrm{N}=4)$ followed by dissection of the nerve from cervical level to below the pulmonary branches, including the cardiac and laryngeal branches, for ex vivo microCT. (B) Identification of the areas responsible for cardiac (C), pulmonary (P) and laryngeal (L) functions in the cervical vagus nerve with EIT. The color scale is arbitrary units $(Z$ score of relative change in the modulus of the impedance). (C) Selective stimulation through individual electrode pairs applied sequentially around the circumference of the cuff (pairs 1-14) with resulting physiological changes, such as heart rate (HR, red, cardiac), electromyography (EMG, green, recurrent laryngeal) and end-tidal carbon dioxide (EtCO2, blue, pulmonary), to determine cross-sectional location of the fascicular groups responsible for the respective functions. (D) MicroCT scanning of the full dissected vagus nerve followed by segmentation and tracing from the point of organ-specific branching up to the cervical level of cuff placement to identify the location of organ-specific fascicles (cardiac, red; laryngeal, green; and pulmonary, blue).

\section{Results}

\section{Functional imaging of the fascicular organization of the vagus nerve in vivo}

20 Techniques allowing imaging of the anatomy of peripheral nerve in vivo include photoacoustic tomography, magnetic resonance imaging (13), optical coherence tomography (OCT) (14-18) and ultrahigh-frequency and high-resolution ultrasound (19-21). Unfortunately, none have sufficient tissue contrast, resolution, clarity and penetration depth to trace fascicles confidently along the entire length of the vagus nerve which is $>60 \mathrm{~cm}$ in large animals such as the pig or humans.

\section{EIT allows imaging of organ-specific activity within the vagus nerve}

Electrical Impedance Tomography (EIT) is a method that can be used to image and identify organspecific fascicles within the vagus nerve by correlation of electrical compound action potentials (CAPs) within the nerve with spontaneous rhythmical physiological activity, such as the heartbeat, lung inflation/deflation, or bowel movement (8). EIT enables the production of images of the internal electrical impedance of a subject using external electrodes. Fast Neural EIT (FN-EIT) enables imaging of neuronal activity within brain or nerve by the detection of small variations in electrical impedance produced by the opening of ion channels during firing and the consequent decrease in membrane resistivity. In peripheral nerves, FN-EIT is performed with a circumferential electrode array set on a nerve cuff, and thus is non-penetrating. It has been demonstrated in rat sciatic nerve with a resolution of $1 \mathrm{msec}$ and $<200 \mu \mathrm{m}(22-25)$. The cuff contained 14 electrodes, each $1.50 \times 0.35 \mathrm{~mm}$, arranged radially around the long axis of the nerve. EIT images were produced from 196 transfer impedances. Each was made by injection of a constant current of 200 $\mu \mathrm{A}$ at $6 \mathrm{kHz}$ to a pair of electrodes spaced 5 electrodes apart and demodulated voltages recorded at all 14 electrodes with respect to a reference ring electrode around the nerve. The current was moved sequentially to all available 14 radial positions. EIT images were then produced using a 2.5M element finite element cylindrical model and represent the impedance decrease related to ion channel opening during the compound action potential in activated fascicles. FN-EIT imaging was performed to image evoked neural activity from the recurrent laryngeal nerve and spontaneous neural traffic from vagal pulmonary and cardiac branches. FN-EIT of spontaneous activity in the nerve was undertaken by coherent averaging of recorded signals in relation to rhythmic physiological activity such as respiration, ECG, or electrical stimulation of the recurrent laryngeal nerve at $20 \mathrm{~Hz}$ with a stimulating electrode in the laryngeal muscle at the same level as cuff 
placement. A SNR of $4.1 \pm 1.5,3.1 \pm 0.16$, and $3.1 \pm 0.65$ with $120 \pm 32,126 \pm 22,126 \pm 22 \delta \mathrm{V}$ traces $(\approx 83,87$ and $87 \%$ of available) were used for image reconstruction for EIT of recurrent laryngeal, pulmonary, and cardiac activity respectively. These revealed distinct regions corresponding to the three functions (Fig. 2C). Locations of peak functional activity identified by FN-EIT matched postmortem microCT tracing with an accuracy of $<25 \%$ of nerve diameter, equally split between the radial and angular component.

\section{Selective stimulation allows for localization of organ-specific activity}

Spatially-selective stimulation was undertaken with consecutive stimulation of a pair of electrodes in the same radial positions in two rings of 14 electrodes spaced $4 \mathrm{~mm}$ apart around the nerve $(8$, $26,27)$ and recording of changes in physiological parameters such as heart rate, breathing frequency, or laryngeal EMG) as an index of evoked end organ activity. Supramaximal stimulation was achieved with $0.4-0.8 / 1-2 / 0.1-0.2 \mathrm{~mA}, 50 \mu \mathrm{s} / 1 \mathrm{~ms} / 50 \mu$ s pulse width, at $20 \mathrm{~Hz}$ for pulmonary, cardiac and laryngeal stimulation, respectively. This caused significant changes of $-38.6 \pm 13.7 \%$, $-10.1 \pm 4.4 \%$, and an increase of $>10 \mathrm{x}$ baseline in the ECG, respiratory frequency, and EMG RMS value, respectively. In each case, $2-4 / 14$ of the serial radial electrode pair sites elicited significantly greater changes in peripheral activity (Fig. 2A).

\section{MicroCT as a gold standard for imaging structural fascicular organization}

Micro-computed tomography (microCT) of peripheral nerve after iodine staining allows ex vivo $3 \mathrm{D}$ tomographic imaging of the vagus nerve with a spatial resolution of $\approx 4 \mu \mathrm{m}$. This method has been developed and validated for the task of the tracing of fascicles over tens of $\mathrm{cm}$ from the innervated organ neural stimulation site to the cervical level in large animals such as the pig or man. This provides independent anatomical validation of any functional connections identified with FN-EIT and selective electrical stimulation (28).

MicroCT was used to image the vagus nerve from the cervical level to distal cardiac, recurrent laryngeal, and pulmonary branches. Lengths of up to $28 \mathrm{~cm}$ could be imaged with an isotropic voxel size of $7 \mu \mathrm{m}$, digitally aligned between overlapping regions between scans, and segmented and traced with Neurolucida 360 (Version 2021.1.3, MBF Bioscience LLC, Williston, VT USA) $(\mathrm{N}=4,24.25 \mathrm{~cm} \pm 1.66$ in length) (Fig. 2B, D, F). There was clear distinguishability of the fascicles from the rest of the nerve soft tissue (28). FN-EIT and selective stimulation were undertaken with different cuffs at one level each, 3 and $5.5 \mathrm{~cm}$ inferior to the nodose ganglion, respectively (termed "cranial" and "caudal" cuff level); microCT analysis was undertaken at both levels. All contoured and segmented regions identified as fascicles in a cross-section of the nerve from the middle of both cranial and caudal cuff placements were validated against the histology with H\&E and semithin sections (Fig. S1).

\section{Consistent organization of cardiac, pulmonary, and recurrent laryngeal structural and} functional fascicular groups across animals

Functional activity and anatomy for the three end-organs correlated largely exclusively to different regions of the cross-section of the left vagus nerve in the proximal neck. Overall, the cardiac, pulmonary and laryngeal fascicles were located ventromedially, dorsomedially, and ventrolaterally to laterally, respectively, for all three techniques and all four nerves (Fig. 2A-G). There were $30.5 \pm 4.4$ fascicles present per nerve. Of these, there were $1.4 \pm 0.5,17.8 \pm 5.7$ and $15.0 \pm 2.6$ fascicles correlating to cardiac, pulmonary, and laryngeal activity, respectively. The cardiac correlating fascicles were exclusive; $3.6 \pm 2.1$ correlated to both pulmonary and recurrent laryngeal function (mean $\pm \mathrm{SD}$ ). Angular separation with respect to the laryngeal fascicles was 
$118 \pm 37^{\circ}$ and $114 \pm 77^{\circ}$ for EIT, $125 \pm 42^{\circ}$ and $128 \pm 36^{\circ}$ for selective stimulation (SS), $102 \pm 28^{\circ}$ for cranial cuff level microCT, and $146 \pm 22^{\circ}$ and $92 \pm 27^{\circ}$ for caudal cuff level microCT for pulmonary and cardiac, respectively ( $\mathrm{p}<0.05$ except 0.06 for cardiac laryngeal FN-EIT). After clusterization and centers of mass $(\mathrm{CoM})$ analysis, dispersion of fascicles around their center was $408 \mu \mathrm{m}, 361$ $\mu \mathrm{m}, 156 \mu \mathrm{m}$, and $182 \mu \mathrm{m}$ for EIT, SS, cranial and caudal level microCT, respectively (Fig. 2E). The area of the cervical vagus nerve was $3.5 \pm 0.3$ and $2.9 \pm 0.40 \mathrm{~mm}^{2}$, for cranial and caudal cuffs respectively. The areas of the regions containing the correlating fascicular groups was equal and greater for pulmonary and laryngeal and $\approx 10 \mathrm{x}$ smaller for cardiac correlation $(32.4 \pm 5.8,29.4 \pm 3.5$ and $3.5 \pm 1.4 \%$ of total area, respectively). Overlap between pulmonary and laryngeal function occurred in $6.5 \pm 3.8 \%$ of the nerve area containing fascicles $(62.9 \pm 10.1 \%)$. 
bioRxiv preprint doi: https://doi.org/10.1101/2022.02.24.481810; this version posted February 25, 2022. The copyright holder for this preprint (which was not certified by peer review) is the author/funder, who has granted bioRxiv a license to display the preprint in perpetuity. It is made available under aCC-BY 4.0 International license.

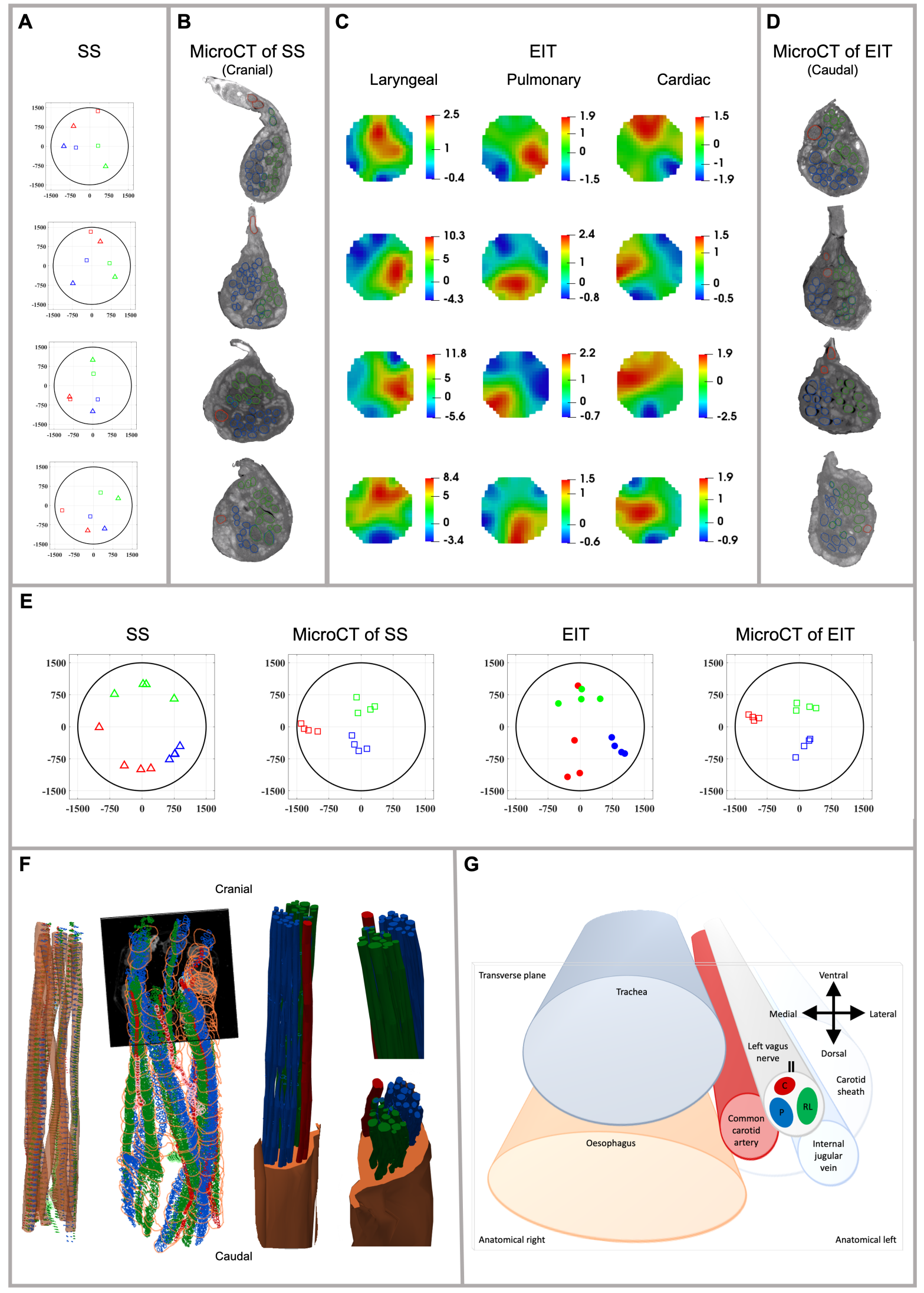


Fig. 2: EIT, SS and microCT results $(\mathrm{N}=4)$ (laryngeal in green, pulmonary in blue and cardiac in red). (A) Selective stimulation (SS) CoM plots. (B) MicroCT cross-sections of the center of SS cuff with segmented fascicles. (C) EIT images for each fascicular group in the nerves. The color scale is arbitrary units ( $Z$ score of relative change in the modulus of the impedance). (D) MicroCT cross-sections of the center of EIT cuff with segmented fascicles. A-D rotated with respect to the cuff opening at 12 o'clock. (E) Clustered CoMs of the fascicular groups of each nerve for each technique rotated with respect to the laryngeal fascicles. (E) 3D shelled segmentation of fascicles in the nerve. (F) Schematic of anatomical location of fascicular regions of the left vagus nerve within the pig with a caudal to cranial view. The opening of the cuff is indicated with a double black line and the fascicular groups have a clockwise order of laryngeal, pulmonary and cardiac. $\mathrm{V}=$ ventral, $\mathrm{VM}=$ ventromedial, $\mathrm{M}=$ medial, $\mathrm{DM}=$ dorsomedial, $\mathrm{D}=$ dorsal, $\mathrm{DL}=$ dorsolateral, $\mathrm{L}=$ lateral, and $\mathrm{VL}=$ ventrolateral.

\section{Discussion}

The three methods have enabled the fascicular organization of the cervical vagus nerve at the level of VNS cuff placement to be deciphered, for the first time. It was possible to identify three spatially separated fascicular groups which correlated with cardiac, pulmonary and laryngeal activity with FN-EIT and selective VNS, and this correlated closely with microCT tracing of the organ branches from their entry into the vagus nerve up to the cervical level $(\mathrm{N}=4, \sim 28 \mathrm{~cm})$. The cervical vagus nerve does indeed appear to be arranged organotopically with respect to these three fascicular groups. These findings were consistent between nerves and the functional and structural imaging techniques. In a cross-section, if recurrent laryngeal is placed at the top (12 o'clock), pulmonary and cardiac follow in clockwise order (c. 5 o'clock and 9 o'clock, respectively).

The vagus nerve innervates the heart, tracheobronchial tree and lungs and the muscles of the larynx in addition to several other organs, including the stomach, esophagus, pancreas, liver and gastrointestinal tract (10). In this study, only cardiac, pulmonary, and recurrent laryngeal fascicular groups were studied. This was because EIT can only be undertaken by imaging differences over time. This requires averaging to a repeated trigger or electrical stimulation to an organ with fast myelinated fibers; the ECG, respiration or laryngeal electrically evoked activity appeared to give the most suitable opportunity. Correlation to subdiaphragmatic organs was not undertaken and is currently in progress. It seems probable that some of the fascicles within the three identified fascicular regions also contained subdiaphragmatic innervation as well. This is because in this work the three identified organs appeared to contribute to $\sim 95 \%$ of observed fascicles. As subdiaphragmatic fibers are about one third of the cross-sectional area and nu number of fascicles of the cervical vagus nerve in pigs as well as humans (6), it seems probable that subdiaphragmatic fibers may share some of the fascicles supporting cardiac and pulmonary function identified in this study.

On the microCT studies, it was possible to identify fascicles but not individual fibers. In this work, it appeared that some peripheral fascicles merged into other fascicles and then these combined fascicles diverged more proximally into two or more. If so, the more proximal divergent fascicles were considered to subserve the function of its parent fascicle and included in subsequent tracing of the fascicular group up the nerve. This was on the hypothesis that fibers pass between fascicles during this cross over. This did not pose any uncertainty for cardiac fascicles which remained distinct throughout. However, this may have led to some false positives or overreporting of proximal fascicles for the other fascicular groups, as the diverging fascicle need not necessarily have subserved the parent function. Without tracing of individual fiber function, this effect could 
not have been definitely characterized, and may have led to an overestimate of fascicles subserved by pulmonary or laryngeal function. In principle, this could have been addressed by fascicle tracing using neural tracers, but no reliable technique is described for the length of nerve characterized in this work in the pig. Only the left vagus nerve was used in this study to correspond with the predominant use of left vagi in clinical VNS applications: the left VNS is reported to cause fewer off-target cardiac effects, when treating epilepsy, including bradycardia or asystole These are hypothesized to be mediated primarily by the right vagus (12). In keeping with somatotopic organization of somatic nerves and organotopic organization of the left vagus nerve and that the left and right vagus provide innervation to the same thoracic end targets, it seems reasonable to suspect that there would be a similar organization of fascicles in the right vagus nerve.

These findings support the hypothesis based on somatic fascicle organization that cervical vagus nerves are organized organotopically. Fascicles are delimited by the perineurium. This is formed by cellular layers and collagen fibers which form a sleeve-like tubular sheath surrounding the fascicles $(29,30)$. Its function is to provide tensile strength and elasticity to the nerve. It acts as a diffusion barrier to irritants and maintains homeostasis of the endoneurium and constant intrafascicular pressure $(29,31)$. Its role in fiber organization is not entirely clear but rather seems to be a convenient envelope that allows for the intermingling or movement of fibers from one fascicle to another whilst inadvertently assisting in the maintenance of fiber organization. In somatic nerves, fascicles appear to maintain a somatotopic organization and may have a simpler parallel cable like or more intermingling plexiform arrangement $(32,33)$. In general, there appears to be discrete somatotopic clustering of fibers distally toward the innervated muscles with intermingling of fibers and fascicles proximally $(2,33-35)$. Tracing of fibers in somatic nerves indicated that, despite some movement of fibers between fascicles, they tended to remain clustered together within the proximal fascicle $(33,36,37)$. Within the ANS, there has been development of methods to enable imaging and subsequent tracing of the fascicular anatomy of the vagus nerve $(20,38)$; however, until now, to our knowledge, no conclusive tracing of fascicles within the ANS has been performed. The arrangement in this study in the pig appears to be both cable-like and plexiform, but with the maintenance of discrete fascicular groupings of the three organs at the cervical level, in a fashion similar to that in somatic nerve but with respect to organs.

These results lend hope to the possibility of use of selective VNS in the future. So far, VNS has been primarily used, and FDA-approved, with unselective whole nerve stimulation, for the treatment of drug-resistant epilepsy and depression (39-43). However, VNS as a therapeutic intervention could be expanded to cardiovascular disorders and heart failure (44-47), lung injury (48-50), asthma $(51,52)$, sepsis $(53,54)$, rheumatoid arthritis $(55,56)$, diabetes $(57-59)$, obesity $(60,61)$, pain management $(62,63)$ and to reduce autoimmunity via anti-inflammatory pathway (64-68). Side effects experienced at present include cough, dyspnea and hoarseness, which can be attributed to activation of the recurrent laryngeal nerve fibers in the vagus nerve $(69,70)$, shortness of breath and bradycardia (71), and even long-term side effects, such as the development of hyperglycemia when stimulating to treat epilepsy (72). The presence of organotopically separated fascicle groups could enable selective VNS and avoidance of off-target effects. The recurrent laryngeal fascicles identified at the cervical level accounted for roughly a half of fascicles present, correlating with previous studies (73). Avoidance of undesired stimulation of vagal outflow to the larynx alone could improve tolerability and efficacy of VNS. Another possibility might lie in activating the cholinergic anti-inflammatory pathway to improve the outcomes of acute respiratory distress syndrome, observed frequently during the current Covid-19 pandemic, whilst avoiding pulmonary cholinergic efferents which have conflicting pro-inflammatory effects (74). 
This work was in the pig; ex vivo microCT studies are in progress in humans in our group to ascertain if human vagal mapping is similar in conjunction with human nerve cuff development. With this, relative mapping and alignment could be performed in vivo using physiological readouts (EMG, ECG, respiratory rate) to confirm the respective positions of the three regions upon performing spatially-selective VNS. This could enable selective stimulation in humans for which there is no current technique for in vivo fascicle or nerve activity visualization. It should be possible to identify mapping of the other organs innervated by the vagus nerve using microCT and anatomical tracing. Functional in vivo determination is more challenging for subdiaphragmatic organs which are supplied only with unmyelinated nerve and lack the clear phasic activity present for heart and lungs used in this study. It may nevertheless be possible to corroborate microCT mapping using phasic adaptive electrical stimulation (75) and selective stimulation in the cervical vagus nerve providing there is an end organ readout, such as venous noradrenaline in the splenic vein.

\section{Conclusions}

The left cervical vagus nerves of pigs were reproducibly organized with respect to cardiac, pulmonary, and recurrent laryngeal function. This supports the hypothesis that fascicles in the autonomic nervous system are organized, at least to a substantial extent, in an organotopic fashion. This supports the possibility of changing current practice in VNS with selective stimulation and increasing therapeutic efficiency by avoidance of off-target effects. The novel techniques of FNEIT and trail-and-error selective stimulation show promise for in vivo imaging of functional fascicular organization and localization clinically in humans with an accuracy sufficient for targeted VNS.

\section{Materials and Methods}

\section{Electrode arrays}

Electrode arrays for selective stimulation and EIT imaging were designed to wrap around pig vagus nerves 2.8-3.0 mm in diameter. Arrays were made from laser cut stainless steel foil (12.5 $\mu \mathrm{m}$ thick) isolated on both sides with medical grade silicone rubber. There were two designs: 1 ) EIT arrays comprised one ring of $141.50 \times 0.35 \mathrm{~mm}$ pads and $29.00 \times 0.47 \mathrm{~mm}$ reference ring electrodes placed at extremities of the cuff, shunted to operate in tripolar mode. Two external reference ring electrodes were present from a template for earlier designs but were unused in this work. 2) Selective stimulation arrays comprised two rings of $143.00 \times 0.35 \mathrm{~mm}$ pads. Exposed electrode areas were roughened to increase surface area and coated with PEDOT:pTS to reduce contact impedance and noise from the electrode-electrolyte interface (76). This yielded impedances of $<1 \mathrm{~K} \Omega$ and $<5^{\circ}$. Cuffs were glued to inner sides of silicone tubing $(2.7 \mathrm{~mm}$ inner, and $4.7 \mathrm{~mm}$ outer diameter) to maintain tubular shape for wrapping around the nerve.

\section{Acute anaesthetized experiments}

The study was performed using Large White domestic female pigs, weighing 60-70 kg. All experimental procedures were ethically reviewed and carried out in accordance with Animals (Scientific Procedures) Act 1986. On the day of the experiment, animals were pre-medicated with ketamine $(20 \mathrm{mg} / \mathrm{Kg})$ and midazolam $(0.5 \mathrm{mg} / \mathrm{Kg})$ administered by intramuscular injection. Fifteen 
minutes after premedication, a $20 \mathrm{G}$ intravenous catheter was placed in the auricular vein. General anesthesia was induced with propofol $(2 \mathrm{mg} / \mathrm{kg}$, i.v.). Animals were intubated with an endotracheal tube, and anesthesia was maintained with sevoflurane vaporized in a 50:50 mixture of oxygen and medical air. A continuous rate infusion of fentanyl $(0.2 \mu \mathrm{g} / \mathrm{kg} / \mathrm{min})$ was started after induction and continued during the whole experimental procedure. After induction of general anesthesia, the animal was positioned in dorsal recumbency. Indwelling catheters were percutaneously placed in both the external jugular veins and one in the femoral artery (for blood pressure and blood gas monitoring) using ultrasonographic guidance. The animal was instrumented with ECG leads and a pulse oximeter. A spirometer was connected to the tracheal tube. The animal was mechanically ventilated using pressure control mode for the duration of the surgery and for most of the experiment, except when selective VNS were applied for identification of pulmonary responses. Between these periods, if required, animals were placed onto mechanical ventilation to restore normal levels of $\mathrm{CO}_{2}$ (between $35-45 \mathrm{mmHg}$ ). Body temperature was maintained using a hot air warming system if necessary. Ringer lactate fluid therapy at a rate of $5 \mathrm{ml} / \mathrm{kg} / \mathrm{h}$ was administered intravenously throughout the procedure. Routine anesthesia monitoring included vital parameters such as electrocardiogram and invasive arterial blood pressure, central venous pressure; end-tidal $\mathrm{CO} 2$ (EtCO2), end-tidal sevoflurane (EtSev), pulse oximetry and core body temperature (via rectal probe). Some of these parameters (arterial blood pressure, central venous pressure, ECG, EtCO2, EtSev) were also digitally recorded using a 16 channel PowerLab acquisition system (ADInstruments) with LabChart 8 software at $2 \mathrm{kHz}$ sampling frequency. Levels of anesthetic were adjusted accordingly by the anesthetist. In some cases, boluses of propofol or fentanyl were used, if required, and noted on the records. After induction of anesthesia and placement in dorsal recumbency, the ventral neck region was clipped and aseptically prepared using chlorhexidinebased solutions, prior to the placement of sterile drapes, leaving only a small window open for accessing the left cervical vagus nerve. Using aseptic technique, a $20 \mathrm{~cm}$ longitudinal skin incision was made using monopolar electrocautery centered immediately to the left of the trachea. The incision was continued through the subcutaneous tissue and the sternohyoideus musculature using a sharp/blunt technique until encountering the carotid sheath and left vagus nerve. A 5-7 cm long segment of the left vagus nerve was circumferentially isolated by blunt dissection to allow placement of SS and EIT electrodes. The electrode cuffs were placed around the nerve by carefully opening the cuffs and sliding the vagus inside it, with the cuff opening facing ventrally. Electrical ground and earth electrodes were inserted into the surgical field. The impedances of the electrodes were $<1 \mathrm{kOhm}$ at $1 \mathrm{kHz}$. The left recurrent laryngeal nerve was identified within the surgical field, and bipolar stimulating electrode (CorTec $\mathrm{GmbH}$, I.D. 1.2-2 $\mathrm{mm}$ ) was placed around it. EMG needles were implanted into laryngeal muscle to record laryngeal effects of selective VNS. The surgical field was then rinsed with sterile saline and the skin temporarily closed using towel clamps. After the round of selective VNS for identification of pulmonary fascicles (section 2.4), the animal was put back on mechanical ventilation and anesthesia was switched to $\alpha$-chloralose (50 mg/kg initial bolus, thereafter $20-35 \mathrm{mg} \cdot \mathrm{kg}^{-1} \cdot \mathrm{h}^{-1}$ i.v.). During a stabilization period (30 min) after anesthesia transition, the rounds of selective VNS to identify the recurrent laryngeal fascicles were performed. Selective stimulation for identification of cardiac branches was performed afterwards, and the remaining 2-3 hours of the experiment were dedicated to spontaneous EIT recordings. At the end of the EIT recordings, an overdose of pentobarbital sodium ( $100 \mathrm{mg} / \mathrm{kg}$ i.v. $)$ followed by saturated $\mathrm{KCl}(1-2 \mathrm{mg} / \mathrm{kg}$ i.v.) was used for euthanasia. 


\section{Fast neural Electrical Impedance Tomography}

Fast Neural EIT imaging to identify laryngeal, cardiac or pulmonary activity was undertaken with averaging triggered from electrical stimulation of the recurrent laryngeal nerve, the ECG or respiration, respectively, with applied current of $6 \mathrm{kHz}$ and $200 \mu \mathrm{A}$ in a skip-5 configuration using a 14-electrode cuff placed $5.5 \mathrm{~cm}$ distal to the nodose ganglion at cervical level. Recorded voltage traces were band-pass filtered with a \pm 1 (larynx) or $2 \mathrm{kHz}$ (cardiac and pulmonary) bandwidth around carrier frequency, demodulated with a Hilbert transform and averaged to improve the Signal-to-Noise Ratio (SNR) before supply to the algorithm for image reconstruction.

Laryngeal EIT was performed using a method previously described for the rat sciatic nerve (2225). Stimulation was undertaken with a bipolar cuff (CorTec $\mathrm{GmbH}$, Freiburg im Breisgau, Germany) over the recurrent laryngeal branch of the left vagus nerve, approximately $40 \mathrm{~cm}$ from the EIT cuff located on the cervical vagus main trunk with biphasic current pulses, $50 \mu \mathrm{s}$ pulse width and $1.2 \mathrm{~mA}$ amplitude at $20 \mathrm{~Hz}$ for $60 \mathrm{~s}$ for each of the 14 injection pairs (total $14 \mathrm{~min}$ ). Some $\delta \mathrm{V}$ traces were excluded from reconstruction set due to technical shortcomings, such as poor electrode contact to the nerve or damaged electrode tracks. Traces were excluded if $>1-4 \mu \mathrm{V}$ noise level or 2-6/4-8 $\mu \mathrm{V}$ for mean/max amplitude, which varied slightly for each experiment (Supplemental Table S2).

Spontaneous EIT - Technical

EtCO2, ECG and BP were recorded. Voltage traces were recorded for a duration of $480 \mathrm{~s}$ for each of the 14 injection pairs, for a total of $112 \mathrm{~min}$. Demodulated voltage traces $(\delta \mathrm{V})$ were high-pass filtered with a cut-off frequency of $\mathrm{Fc}=250 \mathrm{~Hz}$, converted to RMS signals ( $\delta \mathrm{V}$-RMS) with moving average windows of $0.1 \mathrm{~s}$ and $2 \mathrm{~s}$ for pulmonary and cardiac traces, respectively, and subject to coherent averaging over cardiac- or pulmonary-gated cycles. As for laryngeal recordings, exclusion criteria were applied to identify outlier and artefactual $\delta \mathrm{V}$-RMS traces and exclude them from the reconstruction set. Exclusion criteria were:

- Trace amplitude larger than $0.2 \mu \mathrm{V}$.

- Traces with derivative larger than $50 \mu \mathrm{V} / \mathrm{s}$.

- Traces deviating more than 3 standard deviations from group average at any given point.

- Traces deviating more than 3 standard deviations from average Principal Component Analysis (PCA) of the trace group, computed assuming 3-unit vectors.

- Outlier traces characterized a trend over time visibly different from the group ensemble but not falling into the criteria above. Only present in two animals and in negligible amount, 1 out of $144(0.69 \%)$ and 8 out of $144(5.6 \%)$ traces, respectively.

\section{EIT Setup}

All evoked and spontaneous EIT recordings were performed using a modified version of the

ScouseTom EIT system developed within our group (77). Voltage data from the EIT cuff electrodes were sampled in a true parallel configuration at $50 \mathrm{kSamples} / \mathrm{s}$ and 24-bit resolution by 
the EIT system. Modifications to the EIT system performed to improve performance in a surgery room settings included the use of a battery-powered EIT current source (78) to reduce electrical noise and electrical shielding of the recording system and system-to-cuff cabling.

\section{Image Reconstruction}

While spontaneous and evoked EIT recordings underwent different signal processing steps, all post-processed $\delta \mathrm{V}$ traces underwent the same image reconstruction process $(22,24)$ (Fig. 3). A Jacobian matrix $\mathrm{J}$ was obtained from the forward solution (79) to the electrical current distribution problem, computed over a $2.5 \mathrm{M}$-elements tetrahedral model; matrix $\mathrm{J}$ was converted into a coarse hexahedral version of voxel size $150 \mu \mathrm{m}$ and inverted using 0th-order Tikhonov regularization with noise-based voxel correction. For evoked laryngeal EIT, the amplitude of noise-based correction was fixed at $1 \mu \mathrm{V}$ based on commonly observed noise levels of inter-stimuli signal. For RMS traces resulting from spontaneous pulmonary and cardiac EIT, a fixed noise amplitude level of $10 \mathrm{nV}$ was chosen, based on commonly observed levels of $\delta \mathrm{V}$-RMS signal variability around baseline.

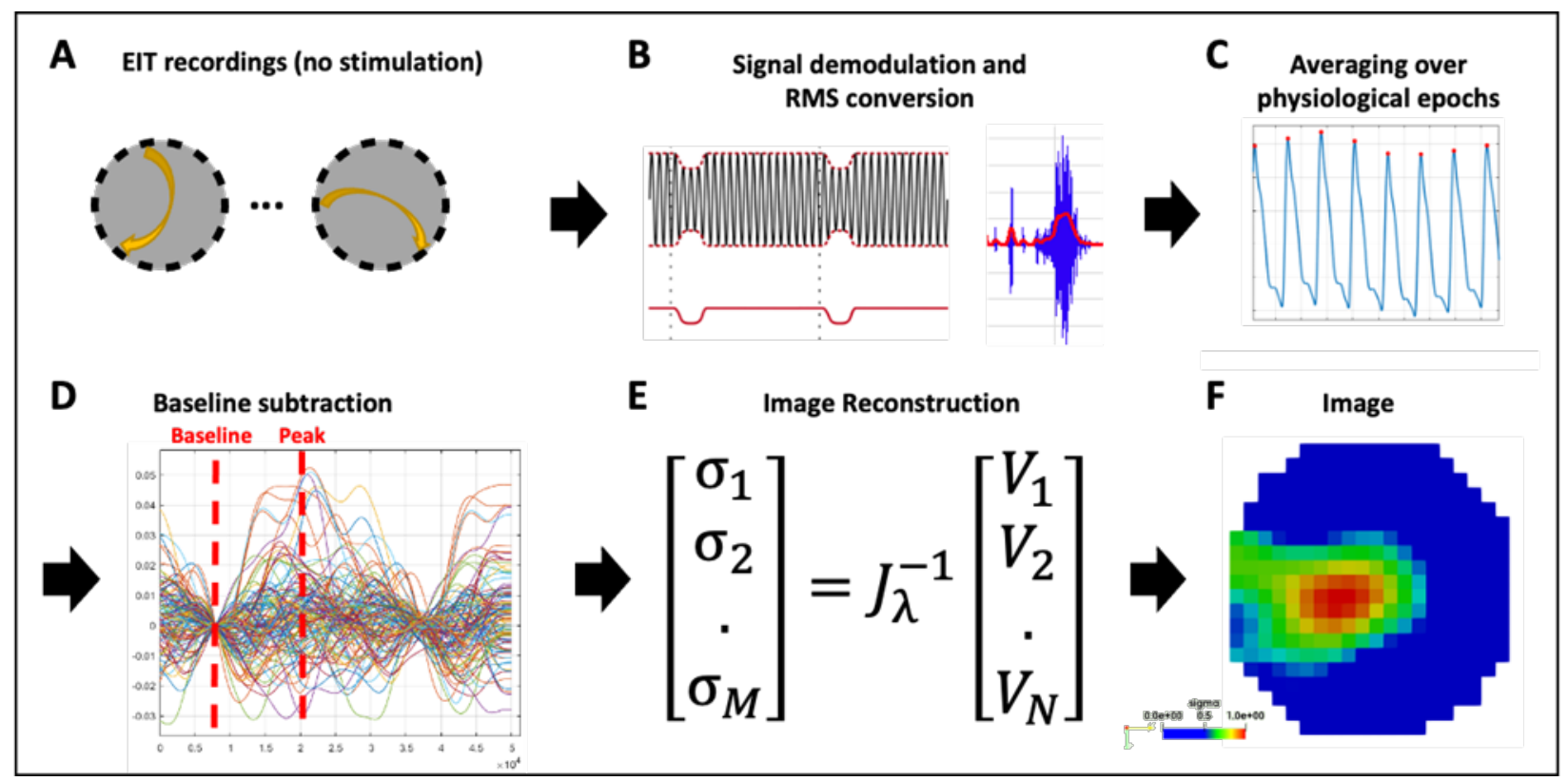

Fig. 3: EIT of spontaneous neural activity. (A) EIT recordings are performed for multiple injection pairs on the cervical vagus nerve cuff. (B) Raw EIT data is demodulated, band-pass filtered and converted to RMS signal ( $\delta \mathrm{V}-\mathrm{RMS})$. (C) Coherent averaging of RMS-converted EIT data is performed over epochs of periodic physiological signals (e.g., heartbeat or breathing). (D) Baseline subtraction is performed over $\delta \mathrm{V}$-RMS traces at a time point corresponding to low or absent neural traffic. (E-F) Tikhonov-based reconstruction is performed to obtain a conductivity map over the cross-section of the nerve. The color scale is arbitrary units ( $Z$ score of relative change in the modulus of the impedance).

\section{Selective vagus nerve stimulation}

Selective neuromodulation was enabled on a cylindrical cuff with two electrode ring arrays by electrical stimulation through a pair in the same radial position on the two rings and cycling 
through all 14 available electrode pairs in a consecutive order. A previous modelling study has demonstrated that this can achieve focused stimulation down to two thirds of the nerve's radius with an angular spread of $26^{\circ}(80)$. Stimulation was applied in pulse trains at $20 \mathrm{~Hz}$ followed by periods of rest of equal duration which were $15 \mathrm{~s}$ or $30 \mathrm{~s}$ for pulmonary and cardiac or $5 \mathrm{~s}$ for laryngeal. For the pulmonary, cardiac, and laryngeal activity, respectively, pulse width (PW) was $50 \mu \mathrm{s}, 1 \mathrm{~ms}$ and $50 \mu \mathrm{s}$ and starting amplitude 400, 1000, and $100 \mu \mathrm{A}$. Pulmonary branch location was assessed by setting ventilation to spontaneous breathing and recording EtCO2 signal as a proxy for breathing rate, while performing selective stimulation on all available electrode pairs. Electrode pair inducing maximum drop-in breathing rate was identified as closest location to pulmonary branch. Stimulation of cardiac branch was identified by pair-selective changes in heart rate (HR) measured from systemic blood pressure (BP) or electrocardiogram (ECG). For this purpose, HR drop was computed as the percentage variation between HR at baseline (no stimulation) and during stimulation at each electrode pair. Electrode pair inducing largest HR percentage drop was classified as location of cardiac branch. Activation of fibers from the recurrent laryngeal branch was assessed by recording needle-based electromyograms (EMGs) from the larynx during selective stimulation and computing the root-mean-square (RMS) signal (band-pass filtering 5-2000 Hz, RMS window $1 \mathrm{~s}$ ). Increase of RMS signal from baseline value indicates larynx activation. Electrode pairs inducing largest RMS increase from baseline were classified as location closest to recurrent laryngeal branches. Images were displayed as Z-scores.

\section{Nerve samples/post-mortem dissection}

Following euthanasia, the left vagi of the animals were dissected from the cervical region (with both the stimulating and EIT electrodes attached) down to the branching regions of cardiac, recurrent laryngeal and pulmonary branches, with all the branches left attached to the main trunk of the vagus nerve. Each sample was approximately $28 \mathrm{~cm}$ in length from the upper cervical level (above electrode cuff placement) to beyond the pulmonary branches at the lower thoracic level. Sutures were placed around the vagus nerve prior to any branching region (i.e., the region where a branch leaves the main vagal trunk) as well as to demarcate the positions of the VNS cuffs. Nerves were then placed in neutral buffered formalin (10\%) for fixation.

\section{MicroCT imaging and segmentation}

\section{Pre-processing and staining}

After fixation, nerve samples were measured, sutures of $1 \mathrm{~cm}$ length were superglued to the vagal trunk in $4 \mathrm{~cm}$ intervals, and nerves cut into $4 \mathrm{~cm}$ lengths at the level of suture placement leaving half of the suture on the end of each section as a marker for subsequent co-registration (Fig. 4). Two to three sections were placed into a tube of $50 \mathrm{ml}$ Lugol's solution (total iodine 1\%; $0.74 \%$ KI, 0.37\% I) (Sigma Aldrich L6141) for five days (120 hours) prior to scanning to achieve maximum contrast between fascicles and the rest of the nerve tissue. On the day of the microCT scan, the nerve was removed from the tube and blotted dry on paper towel to remove any excess Lugol's solution. The nerve sections were placed next to each other onto a piece of cling film (10 $\mathrm{cm} \times 5 \mathrm{~cm}$ ) (Tesco, United Kingdom) in order from cranial to caudal along the length of the nerve, with cranial ends at the top, and sealed with another piece of cling film to retain moisture during the scan as to avoid shrinkage of the nerve tissue. The sealed nerve samples were rolled around a cylinder of sponge $(0.5 \mathrm{~cm} \mathrm{D} \mathrm{x} 4.5 \mathrm{~cm})$ and wrapped in another two layers of cling film to form a 
tightly wound cylinder with a diameter of $\sim 1.5 \mathrm{~cm}$ to fit within the field of view at the required resolution. The wrapped cylinder was placed inside a 3D-printed mount filled with sponge around the edges, ensuring a tight fit and the ends sealed with tape (Transpore ${ }^{\mathrm{TM}}, 3 \mathrm{M}$, United Kingdom).

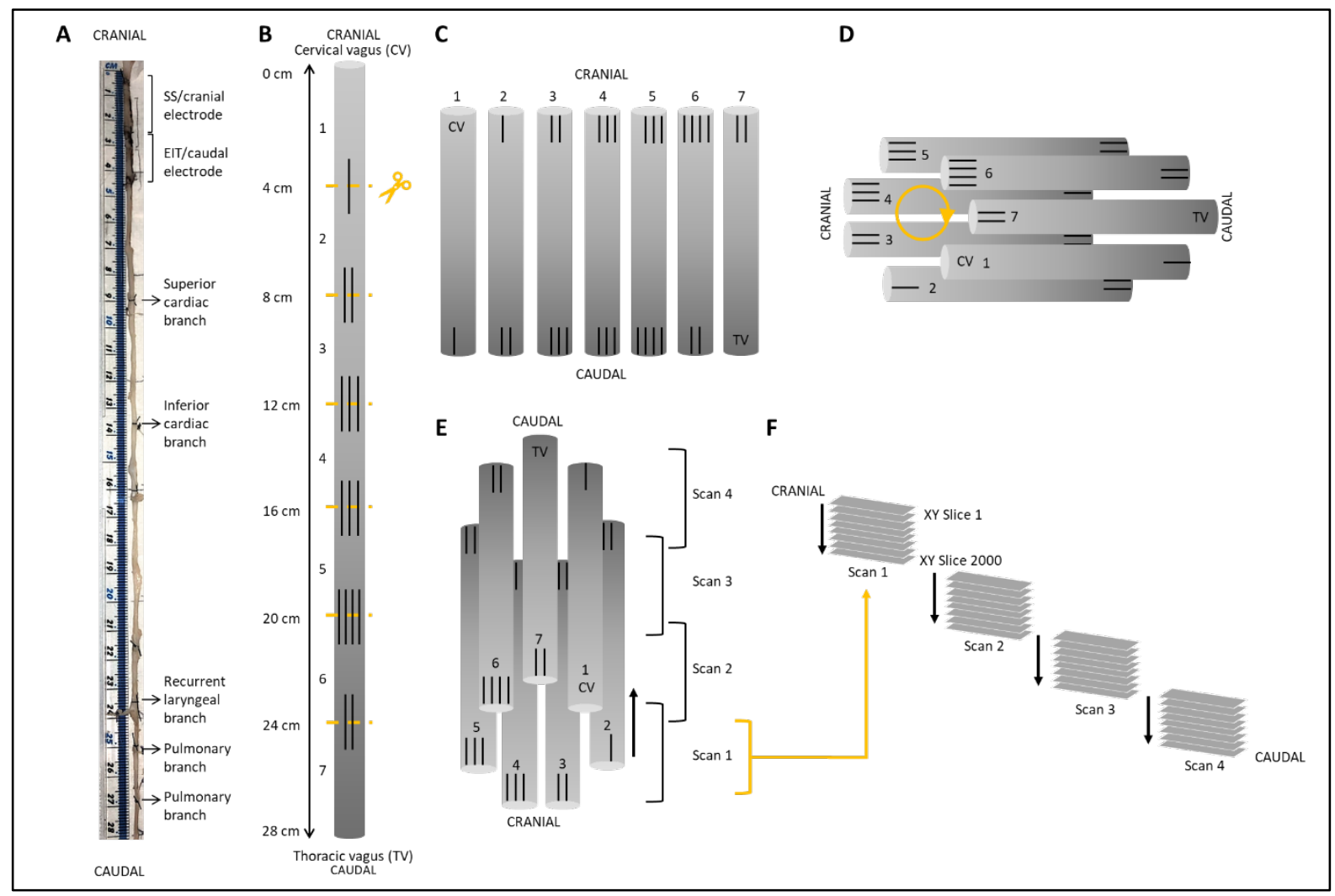

Fig. 4: Schematic of nerve preparation. (A) Photo of a nerve with sutures tied around the vagal trunk proximal to the branching region and demarcating ends of the two electrode cuffs. Each nerve was approximately $28 \mathrm{~cm}$ in length. (B) Sutures were superglued into place every $4 \mathrm{~cm}$ along the length of the nerve with varying numbers to allow for identification of the sections and alignment during segmentation. Sections were cut at the middle of the markers. (C) After contrast staining, nerve sections were ordered from first to last (cervical (CV) to thoracic vagus (TV)), with cranial sides aligned, into cling film and sealed. (D) The sealed nerve sections were rolled around a cylindrical sponge in a clockwise order and wrapped in more cling film to maintain its shape. (E) The cylinder of nerve samples was placed upside down (cranial side down) into a 3D printed mount and placed in the scanner. (F) This setup allowed for the overlapping scans to follow on from another consecutively in the correct order from cranial to caudal along the whole vagus nerve.

\section{MicroCT scanning and reconstruction}

A microCT scanner (Nikon XT H 225, Nikon Metrology, Tring, UK) was homed and then conditioned at $200 \mathrm{kVp}$ for 10 minutes before scanning and the target changed to molybdenum. The scanning parameters were the following: $35 \mathrm{kVp}$ energy, $120 \mu \mathrm{A}$ current, $7 \mathrm{~W}$ power, an exposure of $0.25 \mathrm{fps}$, optimized projections, and a resolution with isotropic voxel size of $7 \mu \mathrm{m}$. Scans were reconstructed in CT Pro 3D (Nikon's software for reconstructing CT data generated by Nikon Metrology, Tring, UK). Centre of rotation was calculated manually with dual slice selection. 
Beam hardening correction was performed with a preset of 2 and coefficient of 0.0 . The reconstructions were saved as 16-bit volumes and triple TIFF 16-bit image stack files allowing for subsequent image analysis and segmentation in various software.

\section{Image analysis, segmentation and tracing}

Reconstructed microCT scan images were analyzed in ImageJ (81) in the XY plane to view the cross-section of the nerve. The vertical alignment of the nerve was positioned so that the crosssectional plane was viewed in the XY stack and the longitudinal plane in the XZ and YZ stacks. This allowed for validation of the scanning protocol, direction of the nerve, and visual analysis of the quality of the image and the distinguishability of the soft tissues - specifically the identification of the fascicles known to exist within the nerve. AVI files were created from ImageJ to enable stack slice evaluation, identification of suture positions and branching locations of the vagus nerve, and as a reference during segmentation. Image stacks (XY plane along the Z-axis) were loaded into Neurolucida 360 (Version 2021.1.3, MBF Bioscience LLC, Williston, VT USA) and image histograms adjusted to optimize visualization of the fascicles when required. Fascicles of the three target organs/functions (namely, cardiac, recurrent laryngeal and pulmonary fascicular groups) were segmented from the rest of the nerve using the Contour mode from the Trace tools. Starting from identification within branches of the vagus nerve, the fascicles were traced through every slice of each scan up the length of the nerve to the cervical region at the level of cuff placement. Contours demarcating the fascicles were placed at regular intervals of 50 to 100 sections and the segmented fascicles labelled accordingly. If fascicles merged with or split into others at a higher frequency, contours were placed at smaller intervals to ensure accurate tracing. While tracing proceeded in the cranial direction, if identified fascicles merged with unlabeled fascicles, the entire new merging fascicle was incorporated and labelled as that fascicular group being traced as it would thereby continue to contain fibers innervating the target organ further up the nerve. If fascicles merged with others already labelled as a fascicular group of interest, it was subsequently labelled as a fascicle containing nerve fibers to both target organs. To continue tracing across cut regions of the nerve, the superglued suture markers and distinct physiological regions or landmarks were used to align the proximal and distal ends of the cut nerves and tracing continued. For visualization of the fully traced nerve, the four overlapping scans were stitched together by aligning the overlapping regions, excluding duplicate images, and forming one large stack of the segmentation data files. Subsequently, this stack was viewed in the 3D Environment and the contours shelled in $3 \mathrm{D}$.

\section{Histology for validation}

Subsequent to scanning, stained nerves were placed back into neutral buffered formalin for a week which allowed for the Lugol's solution to be soaked out. The formalin was refreshed weekly prior to histology. Nerves were cut into two $0.5 \mathrm{~cm}$ segments at the level of each cuff placement. One segment was embedded in paraffin, sectioned at $4 \mu \mathrm{m}$, stained with Hematoxylin and Eosin (H\&E, a routine stain used to demonstrate the general morphology of tissue) (82), and imaged with light microscopy. The other segment was prepared for resin embedding for semi-thin sections and electron microscopy. Identification of histopathological features was performed, and the images of the H\&E and semi-thin sections were then compared to the corresponding slice in the microCT scan of the same nerve for comparison and validation. The presence and number of fascicles 
visualized in the golden standard of histology and electron microscopy was compared to those identified during segmentation of microCT scans to confirm that what was segmented was correct.

\section{Nerve analysis}

In order to calculate the difference in organization and positioning at the cervical level, the following was determined. The order and location of the three fascicular groups of interest within the nerve were analyzed for each nerve and compared between samples; the cuff opening was used as a reference point, being located ventrally. The number of fascicles present in each of the vagus nerves at the level of both cranial and caudal cuff placements was counted for the vagus as a whole, and within each of the fascicular groups namely cardiac, recurrent laryngeal and pulmonary, as well as for the number of 'overlapping' fascicles between groups. The diameter and area of the whole vagus nerve was calculated from the histology images using ImageJ Analyze-Measure function after both the scale was set and the borders of the nerve were segmented. The area of each of the fascicular group regions identified, the overlapping regions, and the total area containing fascicles was calculated as a percentage of the area of the whole nerve from the microCT images. The distance between the cervical level (cuff placement) to the level of branching was calculated for each branch of the three groups/functions. All counts and measurements were compared between nerves.

\section{Image Co-registration, Quantitative Metrics and Statistical analysis}

SNR was computed for EIT recordings as the ratio between average $\delta \mathrm{V}$ (for laryngeal branch EIT) or $\delta \mathrm{V}$-RMS (for pulmonary/cardiac branches) signal at peak variation and baseline/pre-stimulus average noise. The neuromodulation effect for each branch was computed as variation from baseline value evaluated on the most effective electrode pair. The angular distance of pulmonary and cardiac activation peaks from laryngeal were computed for EIT, SS and microCT. Discrimination power was assessed by T-test for each technique. Spatial precision of selective stimulation and localization power of EIT imaging were assessed by comparing center of mass (CoM) locations from the two techniques with CoMs identified from microCT imaging at each cuff. For EIT images, coordinates of peak spontaneous or evoked neural traffic were identified by computing the CoM of the highest-intensity 16 voxels (4x4 area) over the cross-section of the nerve at the time of signal peak. For selective stimulation, focal points of activation for each branch were identified by computing the average radial coordinates of all electrode pairs inducing clearly observable neuromodulation. Selective stimulation currently offers only angular steering capabilities and no depth resolution. Since a radial coordinate is needed to compute cross-sectional coordinates and compare with reference microCT data, peak stimulation was taken to occur at 2/3 of the nerve radius $(80)$. Thus, CoM coordinates were computed assuming peak stimulation at 1 $\mathrm{mm}$ from nerve center, $2 / 3$ of an average $1.5 \mathrm{~mm}$ radius. MicroCT images underwent rigid deformation to generate circular images and were rotated in accordance to cuff opening location to have the same orientation as EIT/SS data. CoM coordinates for each branch were computed by manually labelling the center locations of all fascicles related to a branch and then averaging individual center coordinates. The CoM error over all three branches was first compared in terms of cartesian distance and then split into radial and angular mismatch for easier interpretation. While EIT/SS techniques can be compared to their reference microCT data on individual nerves, grouping of CoM results over multiple nerves to analyze relative distribution of branches poses a challenge due to their different location in relation to the cuff angular orientation. A clusterization 
procedure (83) was adopted for EIT, SS and microCT to perform rigid rotation of all CoM data for each nerve and minimize dispersion of CoM from each branch (Fig. 5). All quantities in this work are reported as mean \pm standard deviation (SD) unless specified.

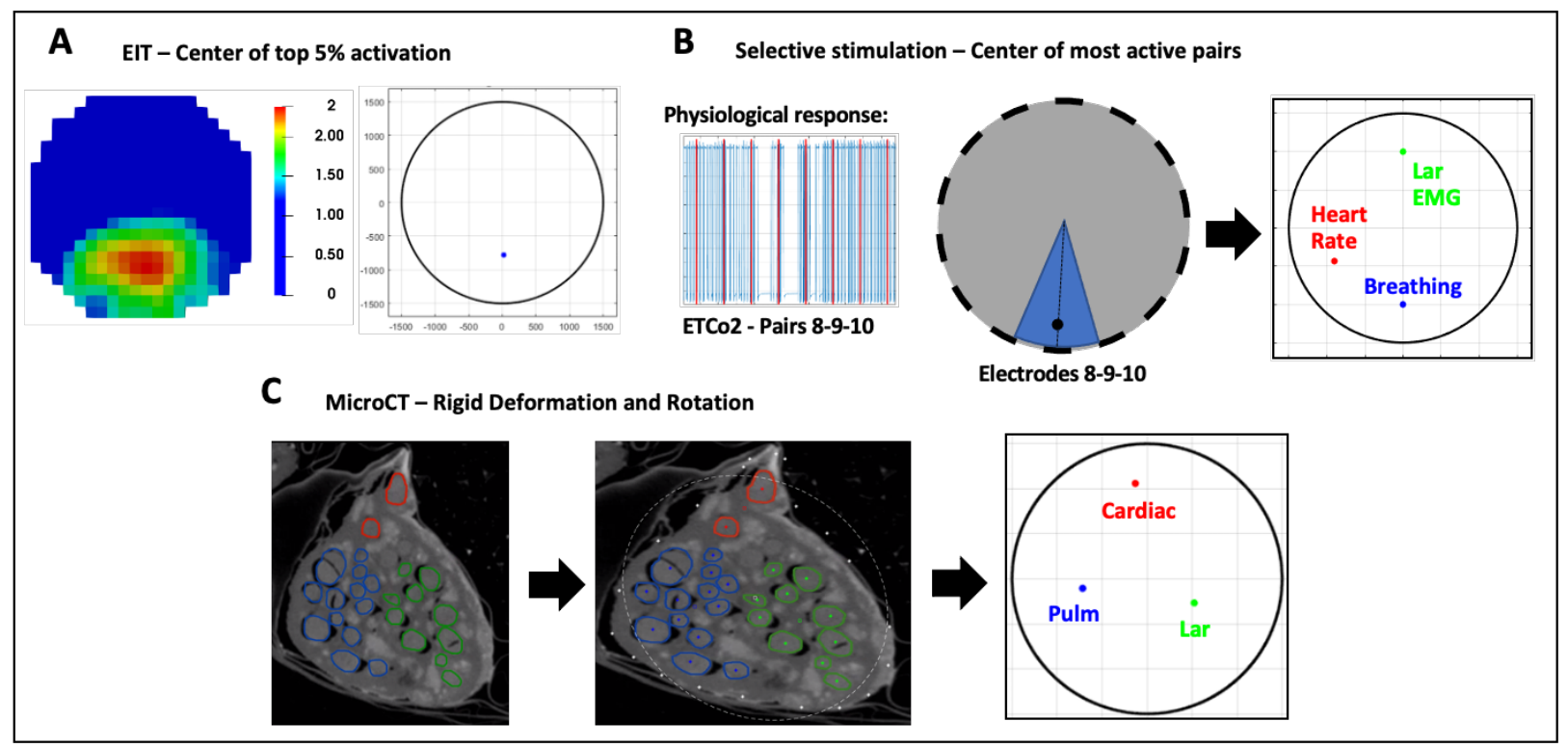

Fig. 5: Image co-registration. (A) Voxels with top 5\% magnitude from the reconstructed EIT image are selected to compute CoM of functional activation. The color scale is arbitrary units $(\mathrm{Z}$ score of relative change in the modulus of the impedance). (B) Electrode pair(s) inducing most localized and highest-intensity physiological response during selective stimulation are chosen to compute angular component of the center of mass for this technique; radial component is chosen as $2 / 3$ rds of nerve radius. (C) Centre of mass for each branch is computed from segmented MicroCT images which are then subject to rigid deformation and rotation for co-registration with the other methods.

\section{References and Notes}

1. P. Bäumer, M. Weiler, M. Bendszus, M. Pham, Somatotopic fascicular organization of the human sciatic nerve demonstrated by MR neurography. Neurology. 84, 1782-1787 (2015).

2. S. Sunderland, The intraneural topography of the radial, median and ulnar nerves. Brain. 68, 243-299 (1945).

3. J. D. Stewart, Peripheral nerve fascicles: Anatomy and clinical relevance. Muscle \& Nerve. 28, 525-541 (2003).

4. N. Hammer, S. Löffler, Y. O. Cakmak, B. Ondruschka, U. Planitzer, M. Schultz, D. Winkler, D. Weise, Cervical vagus nerve morphometry and vascularity in the context of nerve stimulation - A cadaveric study. Scientific reports. 8, 7997 (2018).

5. T. J. M. Verlinden, K. Rijkers, G. Hoogland, A. Herrler, Morphology of the human cervical vagus nerve: Implications for vagus nerve stimulation treatment. Acta Neurologica Scandinavica. 133, 173-182 (2016).

6. N. A. Pelot, G. B. Goldhagen, J. E. Cariello, E. D. Musselman, K. A. Clissold, J. A. Ezzell, W. M. Grill, Quantified Morphology of the Cervical and Subdiaphragmatic Vagus Nerves of Human, Pig, and Rat. Frontiers in Neuroscience. 14, 1148 (2020). 
7. P. Rea, in Clinical Anatomy of the Cranial Nerves, P. Rea, Ed. (Academic Press, San Diego, 2014; http://www.sciencedirect.com/science/article/pii/B9780128008980000105), pp. 105-116.

8. E. Ravagli, S. Mastitskaya, N. Thompson, F. Iacoviello, P. R. Shearing, J. Perkins, A. V. Gourine, K. Aristovich, D. Holder, Imaging fascicular organization of rat sciatic nerves with fast neural electrical impedance tomography. Nature Communications. 11, 6241 (2020).

9. K. Birmingham, V. Gradinaru, P. Anikeeva, W. M. Grill, V. Pikov, B. McLaughlin, P. Pasricha, D. Weber, K. Ludwig, K. Famm, Bioelectronic medicines: a research roadmap. Nature Reviews Drug Discovery. 13, 399 400 (2014).

10. N. Thompson, S. Mastitskaya, D. Holder, Avoiding off-target effects in electrical stimulation of the cervical vagus nerve: Neuroanatomical tracing techniques to study fascicular anatomy of the vagus nerve. Journal of Neuroscience Methods. 325, 108325 (2019).

11. C. M. Ripplinger, From drugs to devices and back again: chemical vagal nerve stimulation for the treatment of heart failure. Cardiovasc Res. 113, 1270-1272 (2017).

12. R. H. Howland, Vagus Nerve Stimulation. Curr Behav Neurosci Rep. 1, 64-73 (2014).

13. G. Rangavajla, N. Mokarram, N. Masoodzadehgan, S. B. Pai, R. V. Bellamkonda, Noninvasive Imaging of Peripheral Nerves. Cells Tissues Organs. 200, 69-77 (2014).

14. D. T. Raphael, C. Yang, N. Tresser, J. Wu, Y. Zhang, L. Rever, Images of Spinal Nerves and Adjacent Structures With Optical Coherence Tomography: Preliminary Animal Studies. The Journal of Pain. 8, 767773 (2007).

15. A. E. Carolus, J. Möller, M. R. Hofmann, J. A. P. van de Nes, H. Welp, K. Schmieder, C. Brenke, Comparison between optical coherence tomography imaging and histological sections of peripheral nerves. Journal of Neurosurgery. 134, 270-277 (2019).

16. A. E. Carolus, M. Lenz, M. Hofmann, H. Welp, K. Schmieder, C. Brenke, High-resolution in vivo imaging of peripheral nerves using optical coherence tomography: a feasibility study. Journal of Neurosurgery. 132, 1907-1913 (2019).

17. S. Vasudevan, J. Vo, B. Shafer, A. S. Nam, B. J. Vakoc, D. X. Hammer, Toward optical coherence tomography angiography-based biomarkers to assess the safety of peripheral nerve electrostimulation. $J$. Neural Eng. 16, 036024 (2019).

18. J. Hope, B. Braeuer, S. Amirapu, A. McDaid, F. Vanholsbeeck, Extracting morphometric information from rat sciatic nerve using optical coherence tomography. JBO. 23, 116001 (2018).

19. R. Beekman, L. H. Visser, High-resolution sonography of the peripheral nervous system - a review of the literature. European Journal of Neurology. 11, 305-314 (2004).

20. M. L. Settell, M. Kasole, A. C. Skubal, B. E. Knudsen, E. N. Nicolai, C. Huang, C. Zhou, J. K. Trevathan, A. Upadhye, C. Kolluru, A. J. Shoffstall, J. C. Williams, A. J. Suminski, W. M. Grill, N. A. Pelot, S. Chen, K. A. Ludwig, bioRxiv, in press, doi:10.1101/2020.12.24.424256.

21. M. S. Cartwright, V. Baute, J. B. Caress, F. O. Walker, Ultrahigh-frequency ultrasound of fascicles in the median nerve at the wrist. Muscle Nerve. 56, 819-822 (2017).

22. K. Aristovich, M. Donegá, C. Blochet, J. Avery, S. Hannan, D. J. Chew, D. Holder, Imaging fast neural traffic at fascicular level with electrical impedance tomography: proof of principle in rat sciatic nerve. Journal of Neural Engineering. 15, 056025 (2018). 
23. E. Ravagli, S. Mastitskaya, N. Thompson, K. Y. Aristovich, D. S. Holder, Optimization of the electrode drive pattern for imaging fascicular compound action potentials in peripheral nerve with fast neural electrical impedance tomography (EIT). Physiological Measurement (2019), doi:10.1088/1361-6579/ab54eb.

24. E. Ravagli, S. Mastitskaya, N. Thompson, F. Iacoviello, P. R. Shearing, J. Perkins, A. V. Gourine, K. Aristovich, D. Holder, Imaging fascicular organization of rat sciatic nerves with fast neural electrical impedance tomography. Nature Communications. 11, 1-10 (2020).

25. E. Ravagli, S. Mastitskaya, N. Thompson, E. J. Welle, C. A. Chestek, K. Aristovich, D. Holder, Fascicle localisation within peripheral nerves through evoked activity recordings: A comparison between electrical impedance tomography and multi-electrode arrays. Journal of Neuroscience Methods. 358, 109140 (2021).

26. K. Aristovich, M. Donega, C. Fjordbakk, I. Tarotin, C. A. R. Chapman, J. Viscasillas, T.-R. Stathopoulou, A. Crawford, D. Chew, J. Perkins, D. Holder, Model-based geometrical optimisation and in vivo validation of a spatially selective multielectrode cuff array for vagus nerve neuromodulation. Journal of Neuroscience Methods. 352, 109079 (2021).

27. E. Ravagli, S. Mastitskaya, N. Thompson, K. Y. Aristovich, D. S. Holder, Optimization of the electrode drive pattern for imaging fascicular compound action potentials in peripheral nerve with fast neural electrical impedance tomography (EIT). Physiol. Meas. (2019), doi:10.1088/1361-6579/ab54eb.

28. N. Thompson, E. Ravagli, S. Mastitskaya, F. Iacoviello, K. Aristovich, J. Perkins, P. R. Shearing, D. Holder, MicroCT optimisation for imaging fascicular anatomy in peripheral nerves. Journal of Neuroscience Methods. 338, 108652 (2020).

29. David J Magee, R. C. Manske, in Orthopedic Physical Assessment (Elsevier, ed. 7, 2021; https://www.clinicalkey.com/\#!/content/book/3-s2.0-B9780323522991000012), pp. 1-72.e2.

30. William K Ovalle, P. C. Nahirney, in Netter's Essential Histology (Elsevier, ed. 3, 2021), pp. 109-139.

31. S. Peltonen, M. Alanne, J. Peltonen, Barriers of the peripheral nerve. Tissue Barriers. 1, e24956 (2013).

32. S. Sunderland, Nerves and nerve injuries (Edinburgh : Churchill Livingstone, Edinburgh, 2nd ed., 1978).

33. M. E. Jabaley, W. H. Wallace, F. R. Heckler, Internal topography of major nerves of the forearm and hand: A current view. The Journal of Hand Surgery. 5, 1-18 (1980).

34. J. N. Langley, M. Hashimoto, On the suture of separate nerve bundles in a nerve trunk and on internal nerve plexuses. J Physiol. 51, 318-346 (1917).

35. J. C. McKINLEY, THE INTRANEURAL PLEXUS OF FASCICULI AND FIBERS IN THE SCIATIC NERVE. Archives of Neurology \& Psychiatry. 6, 377-399 (1921).

36. J. A. Chow, A. L. Van Beek, D. L. Meyer, M. C. Johnson, Surgical significance of the motor fascicular group of the ulnar nerve in the forearm. J Hand Surg Am. 10, 867-872 (1985).

37. G. P. Watchmaker, C. A. Gumucio, R. E. Crandall, M. A. Vannier, P. M. Weeks, Fascicular topography of the median nerve: a computer based study to identify branching patterns. J Hand Surg Am. 16, 53-59 (1991).

38. C. Kolluru, A. Subramaniam, Y. Liu, A. Upadhye, M. Khela, L. Druschel, F. Fereidouni, R. Levenson, A. Shoffstall, M. Jenkins, D. L. Wilson, in Three-Dimensional and Multidimensional Microscopy: Image Acquisition and Processing XXVIII (International Society for Optics and Photonics, 2021; https://www.spiedigitallibrary.org/conference-proceedings-of-spie/11649/1164910/3D-imaging-of-the-vagusnerve-fascicular-anatomy-with-cryo/10.1117/12.2577037.short), vol. 11649, p. 1164910. 
39. C. B. Nemeroff, H. S. Mayberg, S. E. Krahl, J. McNamara, A. Frazer, T. R. Henry, M. S. George, D. S. Charney, S. K. Brannan, VNS Therapy in Treatment-Resistant Depression: Clinical Evidence and Putative Neurobiological Mechanisms. Neuropsychopharmacol. 31, 1345-1355 (2006).

40. J. P. O'Reardon, P. Cristancho, A. D. Peshek, Vagus Nerve Stimulation (VNS) and Treatment of Depression: To the Brainstem and Beyond. Psychiatry (Edgmont). 3, 54-63 (2006).

41. A. H. Young, M. F. Juruena, R. De Zwaef, K. Demyttenaere, Vagus nerve stimulation as adjunctive therapy in patients with difficult-to-treat depression (RESTORE-LIFE): study protocol design and rationale of a realworld post-market study. BMC Psychiatry. 20, 471 (2020).

42. B. Fisher, J. A. DesMarteau, E. H. Koontz, S. J. Wilks, S. E. Melamed, Responsive Vagus Nerve Stimulation for Drug Resistant Epilepsy: A Review of New Features and Practical Guidance for Advanced Practice Providers. Frontiers in Neurology. 11, 1863 (2021).

43. E. Franzoni, V. Gentile, M. C. Colonnelli, D. Brunetto, I. Cecconi, L. Iero, F. C. Moscano, D. M. Cordelli, V. Marchiani, VNS in drug resistant epilepsy: preliminary report on a small group of patients. Italian Journal of Pediatrics. 36, 30 (2010).

44. G. B. Lim, Vagal nerve stimulation in chronic heart failure. Nat Rev Cardiol. 13, 312-312 (2016).

45. Z. U. Asad, S. Stavrakis, Vagus nerve stimulation for the treatment of heart failure. Bioelectronics in Medicine. 2, 43-54 (2019).

46. A. J. Camm, I. Savelieva, Vagal nerve stimulation in heart failure. European Heart Journal. 36, 404-406 (2015).

47. H. U. Klein, G. M. D. Ferrari, Vagus nerve stimulation: A new approach to reduce heart failure. Cardiol J. 17, 638-644 (2010).

48. M. J. Krzyzaniak, C. Y. Peterson, G. Cheadle, W. Loomis, P. Wolf, V. Kennedy, J. G. Putnam, V. Bansal, B. Eliceiri, A. Baird, R. Coimbra, Efferent vagal nerve stimulation attenuates acute lung injury following burn: The importance of the gut-lung axis. Surgery. 150, 379-389 (2011).

49. G. Levy, J. E. Fishman, D. Xu, W. Dong, D. Palange, G. Vida, A. Mohr, L. Ulloa, E. A. Deitch, Vagal nerve stimulation modulates gut injury and lung permeability in trauma-hemorrhagic shock. J Trauma Acute Care Surg. 73, 338-342 (2012).

50. S. Li, D. Qi, J. Li, X. Deng, D. Wang, Vagus nerve stimulation enhances the cholinergic anti-inflammatory pathway to reduce lung injury in acute respiratory distress syndrome via STAT3. Cell Death Discov. 7, 1-9 (2021).

51. E. Steyn, Z. Mohamed, C. Husselman, Non-invasive vagus nerve stimulation for the treatment of acute asthma exacerbations - results from an initial case series. Int J Emerg Med. 6, 7 (2013).

52. S. E. Mehmed, Effect of vagal stimulation in acute asthma. Clin Transl Allergy. 5, P13 (2015).

53. B. Joseph, G. Shimojo, Z. Li, M. del R. Thompson-Bonilla, R. Shah, A. Kanashiro, H. C. Salgado, L. Ulloa, Glucose Activates Vagal Control of Hyperglycemia and Inflammation in Fasted Mice. Sci Rep. 9, 1012 (2019).

54. Z. U. A. Asad, A. Przebinda, A. M. D. Chaudhary, S. Farooqui, H. Youness, S. Stavrakis, The role of vagus nerve stimulation in sepsis. Bioelectronics in Medicine. 3, 51-62 (2020). 
55. SetPoint Medical Corporation, "Vagus Nerve Stimulation Using the SetPoint System for Moderate to Severe Rheumatoid Arthritis: The RESET-RA Study" (Clinical trial registration NCT04539964, clinicaltrials.gov, 2021), (available at https://clinicaltrials.gov/ct2/show/NCT04539964).

56. S. Marsal, H. Corominas, J. J. de Agustín, C. Pérez-García, M. López-Lasanta, H. Borrell, D. Reina, R. Sanmartí, J. Narváez, C. Franco-Jarava, C. Peterfy, J. A. Narváez, V. Sharma, K. Alataris, M. C. Genovese, M. C. Baker, Non-invasive vagus nerve stimulation for rheumatoid arthritis: a proof-of-concept study. The Lancet Rheumatology. 3, e262-e269 (2021).

57. J. Yin, F. Ji, P. Gharibani, J. D. Chen, Vagal Nerve Stimulation for Glycemic Control in a Rodent Model of Type 2 Diabetes. Obes Surg. 29, 2869-2877 (2019).

58. A. M. Drewes, "Treatment of Complications to Diabetic Autonomic Neuropathy With Vagus Nerve Stimulation" (Clinical trial registration NCT04143269, clinicaltrials.gov, 2021), (available at https://clinicaltrials.gov/ct2/show/NCT04143269).

59. E. E. Meyers, A. Kronemberger, V. Lira, K. Rahmouni, H. M. Stauss, Contrasting effects of afferent and efferent vagal nerve stimulation on insulin secretion and blood glucose regulation. Physiol Rep. 4, e12718 (2016).

60. G. Yao, L. Kang, J. Li, Y. Long, H. Wei, C. A. Ferreira, J. J. Jeffery, Y. Lin, W. Cai, X. Wang, Effective weight control via an implanted self-powered vagus nerve stimulation device. Nat Commun. 9, 5349 (2018).

61. G. de Lartigue, Role of the vagus nerve in the development and treatment of diet-induced obesity. $J$ Physiol. 594, 5791-5815 (2016).

62. K. Chakravarthy, H. Chaudhry, K. Williams, P. J. Christo, Review of the Uses of Vagal Nerve Stimulation in Chronic Pain Management. Curr Pain Headache Rep. 19, 54 (2015).

63. University of Medicine and Dentistry of New Jersey, "A Pilot Study to Assess the Tolerability and Exploratory Efficacy of Vagus Nerve Stimulation (VNS) Using the VNS Therapy System in Patients With Refractory Fibromyalgia With and Without Major Depression" (Clinical trial registration NCT00294281, clinicaltrials.gov, 2011), (available at https://clinicaltrials.gov/ct2/show/NCT00294281).

64. B. Bonaz, V. Sinniger, S. Pellissier, Anti-inflammatory properties of the vagus nerve: potential therapeutic implications of vagus nerve stimulation. J Physiol. 594, 5781-5790 (2016).

65. K. J. Tracey, The inflammatory reflex. Nature. 420, 853-859 (2002).

66. L. V. Borovikova, S. Ivanova, M. Zhang, H. Yang, G. I. Botchkina, L. R. Watkins, H. Wang, N. Abumrad, J. W. Eaton, K. J. Tracey, Vagus nerve stimulation attenuates the systemic inflammatory response to endotoxin. Nature. 405, 458-462 (2000).

67. B. Bonaz, C. Picq, V. Sinniger, J. F. Mayol, D. Clarençon, Vagus nerve stimulation: from epilepsy to the cholinergic anti-inflammatory pathway. Neurogastroenterol Motil. 25, 208-221 (2013).

68. G. Matteoli, P. J. Gomez-Pinilla, A. Nemethova, M. Di Giovangiulio, C. Cailotto, S. H. van Bree, K. Michel, K. J. Tracey, M. Schemann, W. Boesmans, P. Vanden Berghe, G. E. Boeckxstaens, A distinct vagal antiinflammatory pathway modulates intestinal muscularis resident macrophages independent of the spleen. Gut. 63, 938-948 (2014).

69. E. N. Nicolai, M. L. Settell, B. E. Knudsen, A. L. McConico, B. A. Gosink, J. K. Trevathan, I. W. Baumgart, E. K. Ross, N. A. Pelot, W. M. Grill, K. J. Gustafson, A. J. Shoffstall, J. C. Williams, K. A. Ludwig, Sources of off-target effects of vagus nerve stimulation using the helical clinical lead in domestic pigs. $J$ Neural Eng. 17, 046017 (2020). 
70. E. Ben-Menachem, Vagus nerve stimulation, side effects, and long-term safety. Journal of Clinical Neurophysiology: Official Publication of the American Electroencephalographic Society. 18, 415-418 (2001).

71. D. M. Mulders, C. C. de Vos, I. Vosman, M. J. A. M. van Putten, The effect of vagus nerve stimulation on cardiorespiratory parameters during rest and exercise. Seizure. 33, 24-28 (2015).

72. H. M. Stauss, L. M. Daman, M. M. Rohlf, R. K. Sainju, Effect of vagus nerve stimulation on blood glucose concentration in epilepsy patients - Importance of stimulation parameters. Physiological Reports. 7, e14169 (2019).

73. M. L. Settell, N. A. Pelot, B. E. Knudsen, A. M. Dingle, A. L. McConico, E. N. Nicolai, J. K. Trevathan, J. A. Ezzell, E. K. Ross, K. J. Gustafson, A. J. Shoffstall, J. C. Williams, W. Zeng, S. O. Poore, L. C. Populin, A. J. Suminski, W. M. Grill, K. A. Ludwig, Functional vagotopy in the cervical vagus nerve of the domestic pig: implications for the study of vagus nerve stimulation. J. Neural Eng. 17, 026022 (2020).

74. S. Mastitskaya, N. Thompson, D. Holder, Selective Vagus Nerve Stimulation as a Therapeutic Approach for the Treatment of ARDS: A Rationale for Neuro-Immunomodulation in COVID-19 Disease. Front. Neurosci. 15 (2021), doi:10.3389/fnins.2021.667036.

75. I. Tarotin, K. Aristovich, D. Holder, Model of Impedance Changes in Unmyelinated Nerve Fibers. IEEE transactions on bio-medical engineering. 66, 471-484 (2019).

76. C. A. R. Chapman, K. Aristovich, M. Donega, C. T. Fjordbakk, T.-R. Stathopoulou, J. Viscasillas, J. Avery, J. D. Perkins, D. Holder, Electrode fabrication and interface optimization for imaging of evoked peripheral nervous system activity with electrical impedance tomography (EIT). Journal of Neural Engineering. 16, 016001 (2019).

77. J. Avery, T. Dowrick, M. Faulkner, N. Goren, D. Holder, J. Avery, T. Dowrick, M. Faulkner, N. Goren, D. Holder, A Versatile and Reproducible Multi-Frequency Electrical Impedance Tomography System. Sensors. 17, 280 (2017).

78. T. Dowrick, G. Sato Dos Santos, A. Vongerichten, D. Holder, Parallel, multi frequency EIT measurement, suitable for recording impedance changes during epilepsy. Journal of Electrical Bioimpedance. 6, 37-43 (2015).

79. M. Jehl, A. Dedner, T. Betcke, K. Aristovich, R. Klofkorn, D. Holder, A Fast Parallel Solver for the Forward Problem in Electrical Impedance Tomography. IEEE Transactions on Biomedical Engineering. 62, 126-137 (2015).

80. K. Aristovich, M. Donega, C. Fjordbakk, I. Tarotin, C. A. R. Chapman, J. Viscasillas, T. R. Stathopoulou, A. Crawford, D. Chew, J. Perkins, D. Holder, Model-based geometrical optimisation and in vivo validation of a spatially selective multielectrode cuff array for vagus nerve neuromodulation. Journal of Neuroscience Methods. 352, 109079 (2021).

81. J. Schindelin, I. Arganda-Carreras, E. Frise, V. Kaynig, M. Longair, T. Pietzsch, S. Preibisch, C. Rueden, S. Saalfeld, B. Schmid, J.-Y. Tinevez, D. J. White, V. Hartenstein, K. Eliceiri, P. Tomancak, A. Cardona, Fiji: an open-source platform for biological-image analysis. Nature Methods. 9, 676-682 (2012).

82. D. C. Sheehan, B. B. Hrapchak, Theory and practice of histotechnology (Battelle Press, Columbus, Ohio, 1987).

83. E. Ravagli, S. Mastitskaya, N. Thompson, E. J. Welle, C. A. Chestek, K. Aristovich, D. Holder, Fascicle localisation within peripheral nerves through evoked activity recordings: A comparison between electrical impedance tomography and multi-electrode arrays. Journal of Neuroscience Methods. 358, 109140 (2021). 
84. N. Hammer, S. Löffler, Y. O. Cakmak, B. Ondruschka, U. Planitzer, M. Schultz, D. Winkler, D. Weise, Cervical vagus nerve morphometry and vascularity in the context of nerve stimulation - A cadaveric study. Sci Rep. 8 (2018), doi:10.1038/s41598-018-26135-8.

85. A. K. Boyd, Vagotomy and the anatomic variations in the vagus nerve. The American Journal of Surgery. $\mathbf{7 8}$, 4-14 (1949).

86. R. S. Tubbs, M. Loukas, M. M. Shoja, D. Blevins, R. Humphrey, G. D. Chua, D. R. Kelly, W. J. Oakes, An unreported variation of the cervical vagus nerve: anatomical and histological observations, 3 .

\section{Acknowledgments:}

Thank you to Maci Heal and Shane Baldwin, MBF Bioscience, for their assistance with Neurolucida 360 software, tools, and training. Thank you to David Goodwin, Royal Veterinary College, for his assistance with histology.

\section{Funding:}

Medical Research Council UK grant MR/R01213X/1 (NT, ER, SM, JP, KA, DH)

National Institutes of Health grant 1OT2OD026545-01 (NT, ER, SM, JP, KA, DH)

\section{Author contributions:}

Conceptualization: KA, DH

Methodology: NT, ER, SM

Investigation: NT, ER, SM, JP, KA

Visualization: NT, ER

Funding acquisition: KA, DH

Project administration: NT, ER, SM, KA, DH

Supervision: KA, DH

Writing - original draft: NT, ER, SM

Writing - review \& editing: NT, ER, SM, JP, KA, DH

\section{Competing interests:}

Authors declare that they have no competing interests.

\section{Data and materials availability:}

Data has been uploaded and is in the curation process. It will be available on Pennsieve

Discover (https://discover.pennsieve.io/) with the title "Organotopic Organization of the Porcine Vagus Nerve - MicroCT, EIT and Selective Stimulation”. This will be updated with the DOI in due course. 